\title{
Estrategia del Rendimiento de la Calidad como factor competitivo en Educación a Distancia y Educación Superior en Educación Universitaria.
}

Recibido: Enero $2008 \quad$ revisado: Junio 2008 aceptado: Enero 2009

Quality Performance Strategy As A Competitive Factor In

Distance Education And Higher Education In University Instruction.

Por: Dr. Claudio Rafael Vásquez Martínez' Dr. Víctor Manuel González Romero Dra. María Morfin Otero ${ }^{3}$ Dra. María Candelaria Rodríguez Pérez ${ }^{4}$ Dra. Angélica Contreras Cueva ${ }^{5}$

\section{Resumen:}

Se ve, a lo largo de la evaluación como algunos criterios de rendimiento académico, considerados como los más avanzados en el proceso de aprendizaje, indicadores por lo mismo de mayor cualificación, presentan logros inferiores por parte de los estudiantes de EDI (Educación a Distancia). Esto no es gratuito y tiene origen en la orientación pedagógica de la educación primaria y secundaria preocupada más por informar y desarrollar destrezas elementales, que por generar en sus alumnos capacidades de observación, análisis, síntesis y desarrollo del pensamiento divergente, de conciencia social y de capacidad de liderazgo para orientar el proceso de cambio social.

\section{Palabras Claves:}

proceso de aprendizaje, logros, indicadores, orientación pedagógica, cambio social.

\section{Abstract:}

Throughout assessment, it can be seen how some criteria of academic performance, considered as being the most advanced ones in the process of learning, which are indicators of higher qualification, show low achievements on the part of the Distance Education students, EDI. This is not unwarranted and it originates in the pedagogical orientation of primary and secondary education, which is more interested in informing and developing elementary skills rather than to generate in the learners observation capabilities, analysis, synthesis and development of the divergent thinking, social consciousness and leadership capabilities in order to guide the process of social change.

\section{Key words:}

Learning process, achievements, indicators, pedagogical orientation, social change.

\footnotetext{
${ }^{1}$ Docente Investigador Universidad de Guadalajara (Mexico), Correo de Contacto con el equipo: crvasquezm@yahoo.com ${ }^{2}$ Docente Investigador Universidad de Guadalajara (México) ${ }^{3}$ Docente Investigador Universidad de Guadalajara (México) ${ }^{4}$ Docente Investigador Universidad de Carabobo (Venezuela) ${ }^{5}$ Docente Investigador Universidad de Guadalajara (México)
} 


\section{Introducción}

Este proyecto abordó el problema de la estrategia del rendimiento de la calidad cualitativa del aprendizaje, estableciendo una comparación entre los resultados de éste y los logros generados en la Educación Presencial, referente obligado de esta comparación.

No se puede desconocer la estrecha relación de la educación con los procesos sociales, económicos, culturales y políticos, no solamente al inicio de un programa de este tipo, sino permanentemente. La formación superior tiene como objetivo el logro de un profesional idóneo independientemente de que su procedencia sea la universidad Presencial o a Distancia. Los resultados de ambas modalidades deben ser similares para un desempeño Semejante dentro de similares circunstancias socio-económicas, culturales y políticas.

\section{Delimitacion Del Problema}

Este estudio abarcó el periodo comprendido entre los periodos del A hasta l es decir de 1996 a 2004, quinquenio para el cual se confrontaron los aspectos referentes al rendimiento de la calidad académica del programa a distancia, en una perspectiva de investigación longitudinal, con los programas paralelos de la Educación Presencial en las áreas de: Biología, Matemáticas, Sociología, Historia, Español, Geografía, Literatura ofrecidas por la Universidad. El rendimiento de los estudiantes a Distancia se comparó por centro zonal de la Universidad.

\section{Encuadre Teorico del rendimiento}

\subsection{El Objetivo Social de EDI como base del Rendimiento}

La educación es un proceso social e histórico concreto, lo cual le otorga una significación precisa y funciones específicas en una sociedad determinada. No se puede desconocer, entonces, que toda educación asume el proceso educativo desde una perspectiva adaptativa y conservadora o innovadora y transformadora.

Es fundamental entender la educación como un proceso que desborda los límites de la escuela; esto alerta hacia la dimensionalidad de la educación y su interacción con los centros laborales, culturales, medios de comunicación masiva y demás instituciones de la comunidad en general, puesto que son la sociedad y las instituciones implícitas en ella, las que educan como un todo. Esta concepción se opone al sistema escolar tradicional para dar cabida a un planteamiento mucho más integral que reconocer las posibilidades, incidencias y determinaciones de otros canales educativos. Es válido retomar aquí el objetivo social del programa EDI: La transformación de la vida de la provincia mediante el potencial científico, actitudinal, dialéctico 
que suele conferir la educación superior. (Velásquez Jiménez [1], Chase [2], Fernández [3], Navarro [4], Trybus [5], Zurita [6]).

\subsection{Indicadores Evaluativos de las Dimensiones de Rendimiento}

La verificación del rendimiento de EDI se emprendió con la mira puesta en estas dimensiones que fueron agrupadas en cuatro componentes dentro de los dominios cognoscitivo y afectivo, haciendo una reducción de las taxonomías ampliamente conocidas al respecto (Bloom [7], Gagné [8]).

La efectividad del rendimiento académico cualitativo del programa a distancia, por otra parte, debe mirarse desde dos ángulos diferentes; de su capacidad para producir los logros esperados de la educación superior, dentro del enfoque de calidad mencionado, capacidad que debe compararse con la estrategia presencial pues ésta se constituye en el único referente conocido para ponderar la efectividad relativa de ambas estrategias y desde su capacidad especial para generar atributos propios de la estrategia a distancia, como es el desarrollo de hábitos de estudio independiente, por ejemplo.

En relación con la Educación presencial, referente conocido para estudiar calidad del producto universitario, se fijaron como componentes de calidad los tipos de aprendizaje denominados análisis, síntesis creativa y valoración, para el dominio cognoscitivo y la satisfacción general para el dominio afectivo.

\section{Objetivos de Investigación.}

\subsection{Objetivo General}

El propósito fundamental de este proyecto fue comparar el rendimiento de la calidad en las características del producto académico obtenido por la Facultad de Educación en las modalidades Presencial y a distancia, con miras a observar la competitividad de ésta última.

\subsection{Objetivo Específico.}

Comparar el rendimiento de la calidad académica de las dos modalidades, en términos de calificaciones y tasas de aprobación por materia. 


\section{Diseño Metodologico Y Ejecucion}

\subsection{Variables de Investigación.}

\subsection{Población y Muestra.}

Los planteamientos teóricos expuestos anteriormente demandaron el control de las siguientes variables: Experiencia docente, área o programa académico, centro zonal, niveles cursados, modalidad académica (Presencial o a Distancia), rendimiento académico.

La población estuvo constituida, de un lado, por los estudiantes matriculados en el programa EDI a partir del periodo A en los programas de Biología, Matemáticas, Sociología, Historia, Español, Geografía, Literatura, adscritos a los centros zonales ya señalados, y por otro lado, por los estudiantes presenciales de los programas paralelos de la Universidad. La población abarcó también a profesores presenciales de curso vacacionales y a asesores de Educación a Distancia.

De la población constituida por los estudiantes a Distancia se extrajo una muestra aleatoria y representativa de los tres niveles académicos ya delimitados. Dicha muestra quedó conformada por 329 estudiantes, a quienes se les aplicó un cuestionario sobre diferentes aspectos del Rendimiento Académico mencionados en la sección de variables de evaluación, obteniéndose una tasa de retorno de 254 cuestionarios.

\subsection{Instrumentación.}

En la elaboración de los instrumentos para obtener la información requerida, se partió de los diferentes aspectos señalados en el marco conceptual sobre el objeto de estudio en cuestión, el Rendimiento académico de los estudiantes del programa a Distancia, entendiéndose éste no sólo en términos de productos (calificaciones), sino también en términos del proceso formativo integral y de los tipos de aprendizaje más complejos.

\section{Resultados y análisis.}

\subsection{El Rendimiento Cuantitativo o las Calificaciones.}

Partiendo del objetivo general que hace referencia a la comparación de las características del producto académico obtenido en las dos modalidades, se elaboró un listado de las materias comunes a las dos modalidades (Presencial-No Presencial) servidas por un mismo profesor entre los periodos del A hasta I. 
Las materias desarrolladas por el mismo profesor en las dos modalidades se agruparon luego de acuerdo con su naturaleza curricular en tres áreas: Profesional Docente, Ciencias Sociales y Humanas y Ciencias Puras y Aplicadas. Así mismo, respondiendo al esquema general de la investigación, se correlacionó este criterio con el criterio de niveles académicos, ordenando estas materias en tres niveles: Inicial, intermedio y final, es decir, buscando que correspondiesen a tres etapas del programa.

A partir de éste listado se extrajo una muestra al azar, cuidando que ella fuera representativa para cada nivel curricular y para cada área académica. Se obtuvo un total de diez y ocho materias, las cuales quedaron agrupadas de la siguiente forma:

$\begin{array}{ll}\text { Area Profesional- Docente } & 7 \text { materias } \\ \text { Area de Ciencias Sociales } & 5 \text { materias } \\ \text { Area de Ciencias Puras y } & \\ \text { Aplicadas } & 6 \text { materias }\end{array}$

El Cuadro No. 0 presenta la muestra total de materias dictadas por el mismo profesor en las dos modalidades, incluyendo además el nivel y el nombre del profesor.

CUADRO No. 0

Materias servidas por el mismo profesor en las dos modalidades entre A hasta I.

$\begin{array}{ll}\text { MATERIA } & \text { NIVEL } \\ \text { Area Profesional Docente } & \\ \text { Español I } & \text { Inicial } \\ \text { Español I } & \begin{array}{l}\text { Inicial } \\ \text { Inicial } \\ \text { Psicología Evolutiva } \\ \text { Sociología de la Educación }\end{array} \\ \begin{array}{ll}\text { Intermedia } \\ \text { Sicología Evolutiva }\end{array} & \text { Inicial } \\ \text { Didáctica General } & \text { Final } \\ \text { Historia de la Pedagogía } & \text { Final } \\ \text { Area de las Ciencias Sociales } & \\ \text { Geografía Física I } & \text { Inicial } \\ \text { Introducción a la Literatura } & \text { Inicial } \\ \text { Geografía Física ll } & \\ \text { (Climatología) } & \text { Intermedia } \\ \text { Demografía y Geografía } & \end{array}$


de la Población

Didáctica de las Sociales

Area de Ciencias Puras y Aplicadas

Matemáticas Básicas I

Matemáticas Básicas I

Geometría Vectorial

Álgebra Lineal

Botánica General

Didáctica de las matemáticas
Intermedia

Final

Inicial

Inicial

Inicial

Intermedia

Intermedia

Final

Las calificaciones obtenidas por los estudiantes en las materias señaladas fueron objeto de análisis, con miras a establecer una comparación del Rendimiento Académico en las dos modalidades. El nivel de significación estadística de las diferencias ente las medias se verificó a través de la prueba ty prueba omega cuadrado.

\subsection{Calificaciones.}

Para adelantar los contrastes de rendimiento cuantitativo entre las modalidades Presencial y a Distancia se extrajo aleatoriamente una muestra de cursos servidos entre 1996 y 2004, procurando que en la muestra quedasen representadas las diferentes áreas del conocimiento y que se mantuviesen constantes el profesor, la materia y el periodo en cada contraste.

Los cuadros 1 a 3 presentan diversas elaboraciones a partir del rendimiento cuantitativo, esto es, considerado en términos de calificaciones. En el Cuadro 1 puede observarse, en primer lugar, que el desempeño de la modalidad Presencial (EDP), supera en promedio y significativamente el rendimiento de la modalidad a Distancia (EDI). De 18 contrastes presentados en este cuadro, 9 resultaron favorables a la Educación Presencial a niveles de significancia inferiores al .05 o sea con una alta probabilidad de certeza estadística en los 9 contrastes restantes no aparecieron diferencias significativas. En ningún caso, entonces, se dio rendimiento superior en la modalidad a Distancia. Las diferencias significativas se dieron, por otra parte, en las áreas de sociales, Matemáticas, Literatura y cursos Psicopedagógicos. 


\section{CUADRO 1}

Diferencias de rendimiento academico entre las Modalidades presencial (EDP) y a distancia (EDI), entre el periodo A hasta I, medias, puntajes $T$ y prueba OMEGA cuadrado $W^{2}$.

\begin{tabular}{|c|c|c|c|c|c|c|c|}
\hline$x, s, t, p w^{2}$ & EDP & & EDI & & & & \\
\hline Materias y Semestre & $\mathrm{X}$ & $\mathrm{S}$ & $\mathrm{X}$ & $S$ & $\mathrm{~T}$ & $\begin{array}{l}\text { P IGUAL O EN FAVOR } \\
\text { DE }\end{array}$ & W2 \\
\hline Periodo & & & & & & & \\
\hline Español & 3.87 & .35 & 3.86 & .7 & .02 & N. S. * Igual & \\
\hline Español & 4.48 & .34 & 3.65 & .5 & 5.53 & $<.001 \mathrm{EDP}$ & .51 \\
\hline Sociol. de la Educación E & 3.83 & .29 & 3.21 & .22 & 6.87 & $<.001 \mathrm{EDP}$ & .21 \\
\hline Geometría Vectorial $\quad E$ & 3.51 & .62 & 3.80 & .60 & 1.50 & N.S. Igual & \\
\hline Matemáticas Básicas I & 3.47 & .62 & 2.92 & 1.14 & 1.68 & N.S. Igual & \\
\hline Historia de la Pedagogía $\mathrm{F}$ & 3.87 & .23 & 3.85 & .29 & .33 & N.S. Igual & \\
\hline Geografía Física II & 3.68 & .45 & 2.71 & .51 & 4.6 & $<.001 \mathrm{EDP}$ & .35 \\
\hline Español I & 3.66 & .69 & 3.53 & .82 & .56 & N.S. Igual & \\
\hline Introducción a la LiteraturaF & 3.32 & .91 & 2.56 & .90 & 2.07 & $<.05 \mathrm{EDP}$ & .095 \\
\hline Sicología Evolutiva & 3.46 & .43 & 3.22 & .39 & 2.11 & $<.05 \mathrm{EDP}$ & .020 \\
\hline Matemáticas Básicas I & 3.87 & .47 & 2.97 & .87 & 6.11 & $<.001 \mathrm{EDP}$ & .39 \\
\hline Geografía Física & 3.68 & .2 & 2.78 & .21 & 3.64 & $<.001 \mathrm{EDP}$ & .34 \\
\hline Didáctica General I & 3.98 & .26 & 3.85 & .18 & 1.68 & N.S. Igual & \\
\hline Demografía y Geografía de & 4.14 & .47 & 3.83 & .60 & 1.22 & N.S. Igual & \\
\hline La Población & & & & & & & \\
\hline Español I & 4.47 & .34 & 3.65 & .50 & 5.53 & $<.001 \mathrm{EDP}$ & 41 \\
\hline Sicología Evolutiva & 3.93 & .48 & 2.86 & .68 & 8.39 & $<.001 \mathrm{EDP}$ & 33 \\
\hline Botánica General & 3.52 & .74 & 3.14 & .76 & 1.88 & N.S. Igual & \\
\hline
\end{tabular}

* N.S.: No significativo. 


\section{Cuadro 2}

Diferencias de rendimiento académico entre los cursos vacacionales de la modalidad a distancia y los cursos presenciales regulares

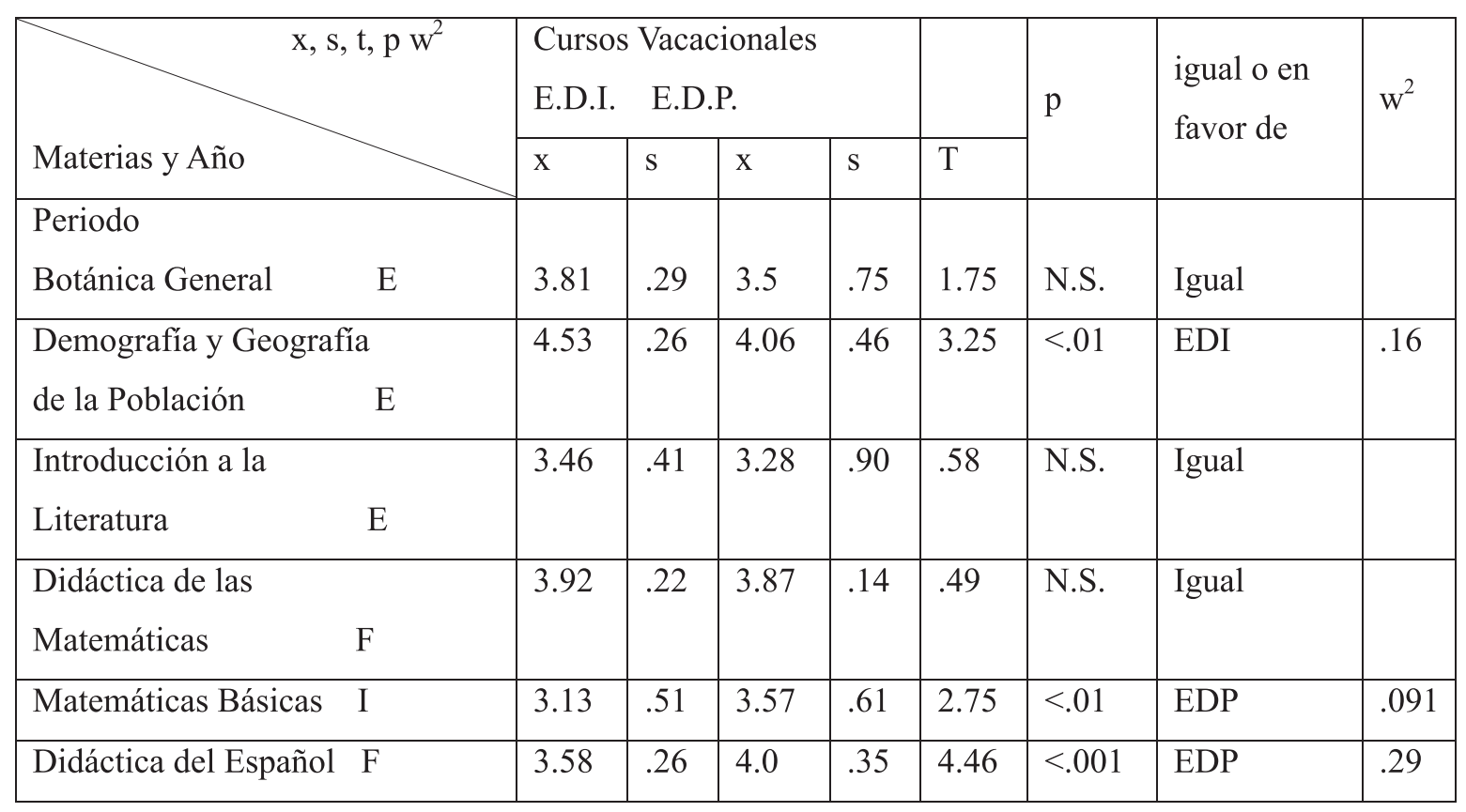

Se aplicó a las diferencias del coeficiente W2, omega cuadrado (Hays [9], [10]), que determina la proporción de la varianza explicada por las variables portadas por las medias contratadas con la prueba $t$, en este caso las modalidades Presencial y a Distancia. El coeficiente fluctuó entre un do $(2 \%$ y un cincuenta y uno $(51 \%)$, explicando en promedio una proporción considerable de la varianza, pero dejando sin explicar otra proporción de ésta debida a factores diferentes a las modalidades.

\subsection{Rendimiento en cursos vacacionales.}

Dado que el rendimiento inferior de los estudiantes a Distancia ha sido atribuido a problemas relacionados con la calidad de las asesorías, con la metodología de materiales e internet, la carencia de bibliotecas regionales, la entrega inoportuna del material y la demora en la información del retorno de trabajos prácticos y evaluaciones en general, problemas no necesariamente consustanciales a la modalidad, sino producto del funcionamiento concreto 0 administración particular de los programas, para verificar la fuerza de estos argumentos, planteados en evaluaciones anteriores y sentidos también en la presente evaluación 
longitudinal, se controló la variable "Cursos vacacionales" servidos en la universidad a finales del año con profesorado universitario, ayudas, orientación e intensidad horaria similares a los de los cursos presenciales. De seis contrastes realizados, uno, Demografía y Geografía de la Población, resultó favorable a EDI a un nivel de significancia inferior también al .05, test no arrojaron diferencias y dos, Matemáticas Básicas y Didáctica del Español, favorecieron a la Educación Presencial, como puede observarse en el Cuadro 2.

Estos resultados apoyan parcialmente la hipótesis de que el rendimiento inferior de los usuarios de EDI tiene que ver con los medios a que están expuestos, primordialmente los módulos y las asesorías, pues al ponérseles en circunstancias mejores en los cursos presenciales, remontan en niveles importantes su rendimiento. Sólo un 33\% de los casos favorecen a la Educación Presencial, pero ya un $16.66 \%$ favorece a la Educación Presencial y un $50 \%$ de los casos arrojan un rendimiento equiparable.

El cuadro 3 complementa o refuerza la tesis anterior. En efecto, al comparar el rendimiento del centro zonal de Guadalajara con la Educación Presencial, el panorama cambia sensiblemente frente al panorama del Cuadro 1. El centro zonal de Guadalajara reúne a los estudiantes de municipios vecinos al Valle de Atemajac. Es lógico pensar, entonces, que estos usuarios pueden trasladarse a la sede central de la universidad en procura de materiales y asesoría individual con mayor frecuencia y facilidad que los estudiantes de los demás centros zonales. El rendimiento mejor del centro zonal de Guadalajara así lo sugiere. Como puede observarse en el Cuadro 3, de los 17 contrastes, sólo 6 favorecen a la Educación Presencial, contra 9 que favorecieron a ésta cuando el contraste fue con todos los centros zonales de la Modalidad a Distancia. El Centro zonal de Guadalajara supera a la Educación Presencial en un contraste, Español I, dándose un rendimiento equiparable en los restantes 10 contrastes. Lo anterior sugiere que los puntajes bajos en calificaciones son aportados en mayor proporción, pues, por los centros zonales diferentes a Guadalajara, cuyos estudiantes no pueden venir con la frecuencia deseable a la universidad y tiene que depender de los módulos, las asesorías, o emisiones radiales y la información que reciben a distancia.

\section{Cuadro 3}

\section{Diferencias de rendimiento académico entre el centro zonal de Guadalajara y la modalidad presencial}

\begin{tabular}{|c|c|c|c|c|c|c|c|c|}
\hline \multirow[b]{2}{*}{ Materias y Semestre } & \multicolumn{2}{|c|}{$\begin{array}{l}\text { EDI } \\
\text { Medellín }\end{array}$} & \multicolumn{2}{|c|}{ E D P } & & \multirow{2}{*}{$\mathrm{p}$} & \multirow{2}{*}{$\begin{array}{l}\text { igual o en } \\
\text { favor de }\end{array}$} & \multirow[t]{2}{*}{$\mathrm{w}^{2}$} \\
\hline & $\mathrm{x}$ & $\mathrm{s}$ & $\mathrm{x}$ & $\mathrm{s}$ & $\mathrm{T}$ & & & \\
\hline Periodo & & & & & & & & .14 \\
\hline Español I & 4.23 & .39 & 3.86 & .35 & 2.31 & $<.05$ & Medellín & \\
\hline
\end{tabular}


Estrategia del Rendimiento de la Calidad como factor competitivo en Educación a Distancia y Educación Superior en Educación Universitaria.

\begin{tabular}{|c|c|c|c|c|c|c|c|c|c|}
\hline Sociol. de la Educación E & & 3.47 & .18 & 3.72 & .09 & -3.14 & $<.001$ & EDP & .06 \\
\hline Geometría Vectorial & $\mathrm{E}$ & 3.42 & .43 & 3.52 & .62 & -.33 & N.S. & Igual & \\
\hline Matemáticas Básicas I & $\mathrm{E}$ & 2.93 & 1.14 & 3.46 & .62 & -1.68 & N.S & Igual & \\
\hline Historia de la Pedagogía $F$ & & 3.63 & .70 & 3.85 & .11 & -1.11 & N.S. & Igual & \\
\hline Álgebra Lineal & $\bar{F}$ & 3.16 & 1.18 & 3.12 & .40 & .06 & N.S. & Igual & \\
\hline Geografía Física II & $\mathrm{F}$ & 3.22 & .40 & 3.70 & .21 & -3.02 & $<.01$ & EDP & .18 \\
\hline Español I & $\mathrm{F}$ & 3.54 & .82 & 3.65 & .69 & -.56 & N.S. & Igual & \\
\hline introd. a la Literatura & $\mathrm{F}$ & 3.26 & .44 & 3.31 & .91 & -.17 & N.S. & Igual & \\
\hline Sicología Evolutiva & $\mathrm{F}$ & 3.23 & .39 & 3.39 & .59 & -1.07 & N.S. & Igual & \\
\hline Matemáticas Básicas I & $\mathrm{F}$ & 2.82 & .58 & 3.83 & .47 & -6.82 & $<.001$ & EDP & .49 \\
\hline Geografía Física & $\mathrm{F}$ & 2.62 & .25 & 3.66 & .20 & -5.20 & $<.001$ & EDP & .52 \\
\hline Didáctica General & G & 3.92 & .07 & 3.96 & .26 & -.98 & N.S. & Igual & \\
\hline $\begin{array}{l}\text { Demog. y Geog.. de la } \\
\text { Población }\end{array}$ & G & 3.84 & .36 & 4.06 & .36 & -.98 & N.S. & Igual & \\
\hline Español I & $\overline{\mathrm{G}}$ & 3.58 & .50 & 4.06 & .34 & -3.17 & $<.001$ & EDP & .13 \\
\hline Sicología Evolutiva & $\mathrm{G}$ & 2.82 & .48 & 4.02 & .19 & -6.59 & $<.001$ & EDP & .26 \\
\hline Botánica General & $\mathrm{H}$ & 3.16 & .78 & 3.5 & .75 & -1.42 & N.S. & Igual & \\
\hline
\end{tabular}

\section{Conclusiones:}

El rendimiento académico de los estudiantes del Programa EDI ha sido inferior en los periodos de A hasta I al rendimiento de los estudiantes de la modalidad presencial. Esta situación se confirmó tanto a través de las contrataciones realizadas a partir de las calificaciones y las tasas de aprobación por materias, como a través de la observación que sobre criterios cualitativos hicieron profesores que han tenido experiencia con alumnos de las dos modalidades.

Estos resultados se derivan en buena parte de las restricciones que afronta el programa en sus aspectos administrativos, financieros y como consecuencia de éstos en su funcionamiento operativo, el cual experimenta serias dificultades en la elaboración cualificada de los materiales didácticas, Internet, en la entrega oportuna de los materiales, en la organización de asesorías de alta calidad, en la suficiente y oportuna retroalimentación al proceso de evaluación y en la organización y dotación mínima de los centros zonales. Todo esto repercute en un aprendizaje de calidad inferior al obtenido en la modalidad presencial que cuenta con mejores condiciones para el ejercicio de la docencia y la calidad de la formación profesional. 
Preocupa una cualificación desventajosa del docente de provincia pues esta cualificación ha sido concebida como elemento de intervención para equilibrar las condiciones generadoras de calidad en los procesos educativo-formativos de los niveles pre-escolar-primaria y secundaría entre la ciudad y la provincia. No se puede esperar que docentes con deficiencias en su formación pedagógica, científica y social, transformen, creen, busquen eficazmente mejores condiciones y alternativas frente a la educación y la cultura de las poblaciones de la Región. Si su formación está en seria desventaja, su proyección actuará en consecuencia.

Se vió a lo largo de la evaluación como algunos criterios de rendimiento académico, considerados como los más avanzados en el proceso de aprendizaje, indicadores por lo mismo de mayor cualificación, presentan logros inferiores por parte de los estudiantes de EDI. Estos no es gratuito y tiene origen en la orientación pedagógica de la educación primaria y secundaria preocupada más por informar y desarrollar destrezas elementales, que por generar en sus alumnos capacidades de observación, análisis, síntesis y desarrollo del pensamiento divergente, de conciencia social y de capacidad de liderato para orientar el proceso de cambio social.

El rendimiento académico de los estudiantes del programa mejora significativamente cuando se ve favorecido el proceso de enseñanza-aprendizaje por las condiciones del ecosistema universitario, hecho que se ha verificado al contrastar las calificaciones de los cursos vacacionales-presenciales de EDI con las de los cursos a distancia y aún con los de la modalidad presencial, lo cual plantea la necesidad de mantener esta combinación hasta tanto mejoren sensiblemente las condiciones culturales y educativas de la provincia. 


\section{Referencias Bibliográficas:}

Velásquez Jiménez (coords.), (1998). La Calidad en la Educación Superior en México. Una Comparacion Internacional; UNAM, México, Chase, Aquilano (1995) Direcciòn y Administración de la Producción y de las Operaciones. Editorial Irwin, sexta edición.

Fernández Díaz, Ma José y González Galán Arturo. (1997). "Desarrollo y Situación Actual de los Estudios de Eficacia Escolar". Revista Electrónica de Investigación y Evaluación Educativa Volumen 3. Número 1_3.

Navarro, Ernesto. (1997). Gestión y Estrategia. № 11 y 12. UAMA-A. Enero - Diciembre.

Trybus, Myron. (1995). Total Quality Management in Schools of Business and Engineering, in Academic Iniciatives in Total Quality for Higher Education, edited by Harry V. Roberts, ASQC Quality Press. Milwaukee, USA.

Zurita Reginaldo (1998), "Crisis de Identidad y de Misión de la Universidad. La Formación Profesional". Artículo publicado en Gestión de la Docencia e Internacionalización de las Universidades Chilenas. CINDA.

Bloom, B. (2001). A taxonomy for learning, Teaching, and assessing: a revison of Bloom's taxonomy of educational objetctives/editors, lorin W. Anderson. David Krathwohl. Complete ed. New York. Longman.

Gagné, R. M. (2005). Principles of instructional design. $5^{\text {th }}$ ed. Belmont. C. A. Thomson/Wadsworth.

Hays, William Lee. (1993). Statistics for psychologistis. New Cork. Holt. Rinehart and Winston.

Hays, William Lee. (1994). Statistics. 5th ed. For Worth: Harcourt Collage Publishers. 